\title{
Fano effect and Andreev bound states in a hybrid superconductor-ferromagnetic nanostructure
}

\author{
E.C. Siqueira $^{\text {a,* }}{ }^{\text {, P.A. Orellana }}{ }^{\text {b }}$, R.C. Cestari ${ }^{c}$, M.S. Figueira ${ }^{\text {d }}$, G.G. Cabrera ${ }^{\text {e }}$ \\ a Departamento de Física, Universidade Tecnológica Federal do Paraná - UTFPR, 84016210, Ponta Grossa, PR, Brazil \\ b Departamento de Física, Universidad Técnica Federico Santa Maria, Av. Vicuña Mackenna 3939, Santiago, Chile \\ c Departamento de Física e Química, Universidade Estadual Paulista - UNESP, 15385-000, Ilha Solteira, SP, Brazil \\ d Instituto de Física, Universidade Federal Fluminense, 24210-340, Niterói, RJ, Brazil \\ e Instituto de Física 'Gleb Wataghin', Universidade Estadual de Campinas - UNICAMP, Campinas 13083-859, SP, Brazil
}

\section{A R T I C L E I N F O}

\section{Article history:}

Received 28 March 2015

Received in revised form 24 July 2015

Accepted 25 July 2015

Available online 29 July 2015

Communicated by L. Ghivelder

\begin{abstract}
A B S T R A C T
In this work, it is considered a hybrid nanostructure composed by a quantum dot coupled to two ferromagnetic leads and a superconductor lead. It is shown that the zero-bias transmittance for the co-tunneling between the ferromagnetic leads presents Fano anti-resonances due to the destructive interference between the two spin channels mixing by the relative orientation of the magnetizations in the leads. When the superconductor is coupled to the system, electron-hole correlations between different spin states lead to a resonance in the place of the dip appearing in the transmittance. Such an effect is accompanied by two Fano anti-resonances explained by a "leakage" of conduction channels from the co-tunneling to the Andreev transport. In the non-equilibrium regime, correlations within the quantum dot introduce a dependence of the resonance condition on the finite bias applied to the ferromagnetic leads. However, it is still possible to observe signatures of the same interference effect in the electrical current.
\end{abstract}

(C) 2015 Elsevier B.V. All rights reserved.

\section{Introduction}

The recent technology of production of quantum dots (QDs) has allowed the implementation of hybrid nanoelectronic devices combining superconductivity and ferromagnetism. In these systems one is able to investigate purely quantum phenomena under the presence of spin polarization and superconducting pair correlations. The electrons are also submitted to Coulomb correlations within the QD. All these features lead to rather complex spectral properties for the QD [1-17]. More specifically, a multi-peak structure has been observed in a F-QD-S nanostructure caused by the interplay between Coulomb correlations and Zeeman splitting due to an external magnetic field [18]. Additionally, regions of negative differential conductance were predicted for a double QD nanostructure coupled to a superconductor and two ferromagnets [19].

In this work, the transport properties of a three-terminal $F_{1}-(Q D, S)-F_{2}$ nanostructure is considered. As illustrated in Fig. 1, the $\mathrm{QD}$ is coupled to two ferromagnets in which a voltage bias is applied to $F_{1}$ while $S$ and $F_{2}$ are kept grounded $\left(V_{2}=0\right)$. The transmittance with the presence of a superconductor coupled to

\footnotetext{
* Corresponding author.

E-mail address: ezcostta@gmail.com (E.C. Siqueira).
}

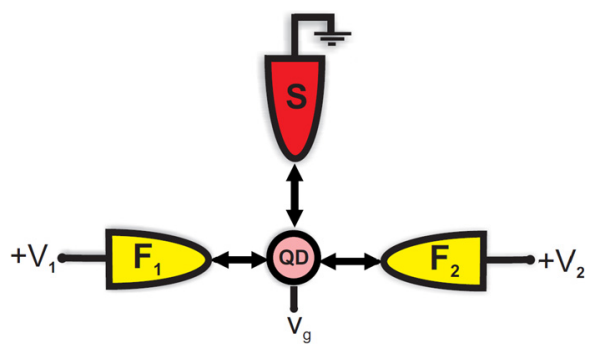

Fig. 1. (Color online.) Schematic diagram for the $F_{1}-(Q D-S)-F_{2}$ system. The magnetization of $F_{1}$ is assumed to be fixed and the magnetization of $F_{2}$ can be varied by an angle $\theta$ with respect to the $F_{1}$ magnetization. $V_{1}$ and $V_{2}$ are the external potentials applied to $F_{1}$ and $F_{2}$, respectively, while the superconductor is grounded. $V_{g}$ is the gate potential applied to the QD.

the QD is studied in order to determine the role of the Andreev reflection (AR) in the co-tunneling current between $F_{1}$ and $F_{2}$. The orientation of the magnetization for the lead $F_{1}$ is fixed while the magnetization of $F_{2}$ is directed to an angle $\theta$ with respect to $F_{1}$. It may be varied from 0 (parallel configuration) to $\pi$ (anti-parallel configuration). The correlations within the QD are treated by using a Hubbard-I approximation [20,21] under which the current still may be expressed in terms of a Landauer-like formula [22]. It worth mentioning that the geometry shown in Fig. 1 has already 
been studied considering the situation in which the QD is noninteracting and addressing non-local transport due to crossed AR $[23,24]$. More recently, spin-dependent conductance and thermoelectric properties were studied for this nanostructure where the role of AR is considered [25-28]. In Ref. [26], the differential conductance and the magnetoresistance have been studied in which a zero-bias anomaly in the Andreev conductance is reported. Such an anomaly is explained by the spin-accumulation generated within the $\mathrm{QD}$ due to the coupling to ferromagnets. In Ref. [27] the authors address the role of the superconductor in the detection of the exchange field induced within the QD due to the coupling to the ferromagnets. More recently, Colci et al. [29] measured the resistance of a multiterminal $\mathrm{S} / \mathrm{F}$ junction finding an anomalous behavior in which the resistance is higher for the anti-parallel configuration of the ferromagnets in contrast to the expected behavior of the crossed AR. This feature was explained by considering spin dependent scattering at the interfaces F/S [30].

Here we focus on the co-tunneling process and how it is affected by the coupling to the superconductor. We observe Fano-like resonances appearing in the transmittance due to the interplay between different spin-channels and the Andreev bound states within the QD. In spite of transport properties have already been addressed in literature [23,31], the Fano-like interference reported here was not at all discussed in previous works. A similar pattern in the transmittance of a double QD coupled to non-magnetic leads [32] was considered but in that case it was a result of Fano interference between different paths for the transport. In contrast, the effect discussed in the present work is a result of an interplay between superconductivity and ferromagnetism which emulates the effect of a Fano interference. In this way, such an effect and the mechanism proposed is original and different from the previous results for $\mathrm{F}_{1}-(\mathrm{QD}, \mathrm{S})-\mathrm{F}_{2}$ system.

This paper is organized as follows: in Section 2 we present the model for the system displayed in Fig. 1 and the physical quantities are determined by using the formalism of non-equilibrium Green's functions. In Section 3 the results are presented and discussed. A summary and the main conclusions are presented in Section 4.

\section{Model and formulation}

In this section we provide a general description of the formalism used to carry out the calculations of the physical quantities. We have used the Keldysh formalism within the Nambu notation $[33,34]$ which allows us to describe spin and electron-hole degrees of freedom in the same footing. This is widely used to tackle systems involving ferromagnets and superconductors.

\subsection{Hamiltonian}

The Hamiltonian for the full system, illustrated in Fig. 1, is written as

$\hat{H}=\hat{H}_{1}+\hat{H}_{2}+\hat{H}_{\mathrm{s}}+\hat{H}_{D}+\hat{H}_{T}$.

The terms $\hat{H}_{1}$ and $\hat{H}_{2}$ are the Hamiltonians describing the ferromagnets $F_{1}$ and $F_{2}$, respectively. Explicitly, we can write:

$\hat{H}_{1}=\sum_{k \sigma} \varepsilon_{1 k \sigma}(0) \hat{a}_{1 k \sigma}^{\dagger} \hat{a}_{1 k \sigma}$

and

$\hat{H}_{2}=\sum_{k \sigma} \varepsilon_{2 k \sigma}(\theta) \hat{a}_{2 k \sigma}^{\dagger} \hat{a}_{2 k \sigma}-\sum_{k \sigma} h_{2} \sin \theta_{2} \hat{a}_{2 k \sigma}^{\dagger} \hat{a}_{2 k \bar{\sigma}}$,

where $\hat{a}_{\eta k \sigma}^{\dagger}$ and $\hat{a}_{\eta k \sigma}$ create an electron and a hole, respectively with spin $\sigma$ and wave-vector $k$ in the ferromagnet $F_{\eta}, \eta=1,2$; $\varepsilon_{\eta k \sigma}(\theta)=\varepsilon_{k}-\sigma h_{\eta} \cos \theta-\mu_{\eta}$ with $\sigma=+(-)$, for spin up (down). The ferromagnets are modeled by the Stoner model [35] in which the spin bands of $F_{\eta}$ are split by an internal mean-field $h_{\eta}$ producing a finite polarization of the electron gas. The magnetization of $F_{1}$ is considered to point to a fixed direction while the magnetization of $F_{2}$ can be rotated by an arbitrary angle $\theta$. The chemical potentials of each ferromagnet are determined by an external voltage bias $\mu_{\eta}=e V_{\eta}$ which controls the Fermi level of each electrode independently.

In addition to the angle $\theta$, an additional phase factor $\phi$ can also appear in a general rotation operation (Euler rotation) on the magnetization of $F_{2}$. However, such a phase factor does not play any role since the up and down spins projections depend only on $\theta$. In fact, it is possible to show that this additional phase is completely eliminated of $\hat{H}_{2}$.

The superconductor is described by

$\hat{H}_{\mathrm{s}}=\sum_{k \sigma}\left(\varepsilon_{\mathrm{s}, k}-\mu_{\mathrm{s}}\right) \hat{a}_{\mathrm{sk \sigma}}^{\dagger} \hat{a}_{\mathrm{sk \sigma}}+\sum_{k}\left[\Delta^{*} \hat{a}_{s k \uparrow}^{\dagger} \hat{a}_{s(-k) \downarrow}^{\dagger}+\right.$ H.c. $]$

where $\varepsilon_{\mathrm{s}, k}=\varepsilon_{k}-\mu_{\mathrm{s}}$, with $\mu_{\mathrm{s}}$ being the superconductor chemical potential which is fixed to zero as the ground $\left(\mu_{\mathrm{s}}=0\right)$. The superconducting correlations enter by means of the pair amplitude $\Delta$ which in general is a complex function depending on $k$. Since we are using just one superconductor lead, we use the wellknown assumption $[23,33,34]$ in which $\Delta$ is just a constant real number. Here we have considered a conventional superconductor (s-wave) being well described by the BCS Hamiltonian [36]. In this case, the ferromagnetism and superconductivity are antagonistic phenomena since the exchange field induces a triplet alignment of spins while the superconductor requires the opposite alignment. This is not always case once the superconductivity also may exist with triplet pairing [37] or even with a mixture of both pairing symmetries [38]. Conversion from singlet to triplet supercurrent is also being studied in half-metallic/superconductor interfaces [39,40] and Josephson junctions [41].

The QD is considered to be interacting with one level degenerated in spin,

$\hat{H}_{D}=\sum_{\sigma}\left(\varepsilon_{0}-e V_{g}\right) \hat{d}_{\sigma}^{\dagger} \hat{d}_{\sigma}+U \hat{d}_{\uparrow}^{\dagger} \hat{d}_{\uparrow} \hat{d}_{\downarrow}^{\dagger} \hat{d}_{\downarrow}$.

We consider that the QD level can be displaced by means of a gate voltage $V_{g}$ with $\varepsilon_{0}$ being the bare QD level (spin degenerated). Hereafter we assume $\varepsilon_{0}=0$ in such a way that the bare $\mathrm{QD}$ level is aligned to the superconductor chemical potential when $V_{g}=0$. The second term describes the Coulomb correlations whose intensity is controlled by $U$; since we are interested in the Andreev regime, $U$ is considered to be smaller than the superconductor gap $\Delta$.

The tunneling between the QD and the leads is described by

$\hat{H}_{T}=\sum_{k \gamma \sigma}\left[t_{\gamma k} \hat{a}_{\gamma k \sigma}^{\dagger} \hat{d}_{\sigma}+\right.$ H.c. $]$

where $\gamma=1,2, s$ and $t_{\gamma k}$ is the tunneling amplitude. Since the energy range is limited to the narrow superconductor gap, it is a good approximation to consider $t_{\gamma k}$ independent on $k$.

\subsection{Green's functions and physical quantities}

In order to calculate the transport properties we have used the non-equilibrium Green's function method [42]. All the physical quantities can be cast in terms of the Green's function of the QD. By using the equation of motion technique [43,44], we are able to determine the equations for these Green's functions in terms of the parameters of the system. A thorough derivation of the Green's functions as well as the corresponding physical quantities may be 
found in Ref. [45]. In the stationary regime, it is possible to write the Green's functions as functions of the energy of the electrons as well as the other parameters of the system. In particular, the coupling strengths between the QD and the leads are given by the constants $\Gamma_{\eta}=2 \pi \mathcal{D}_{\eta}\left(\varepsilon_{F}\right)\left|t_{\eta}\right|^{2}$, with $\eta=1,2$, s. For the ferromagnets, the density of states at the Fermi level is different for spin-up and spin-down electrons. To quantify this, we define a transport polarization for each lead:

$P_{\eta}=\frac{\Gamma_{\eta \uparrow}-\Gamma_{\eta \downarrow}}{\Gamma_{\eta \uparrow}+\Gamma_{\eta \uparrow}}$

where $\eta=1,2$ and $\Gamma_{\eta \sigma}=2 \pi \mathcal{D}_{\eta \sigma}\left|t_{\eta}\right|^{2}$. Instead working with $\Gamma_{\eta \sigma}$, we define an spin average coupling $\Gamma_{\eta}=\left(\Gamma_{\eta \uparrow}+\Gamma_{\eta \downarrow}\right) / 2$ which allows us to write $\Gamma_{\eta \sigma}=\Gamma_{\eta}\left(1+\sigma P_{\eta}\right)$. With these definitions, we work with $P_{\eta}$ and $\Gamma_{\eta}$ as independent parameters.

The physical quantities studied in this work are the electrical current and the transmittance through the QD. The current through the ferromagnet $F_{\eta}$ is defined as the time variation of the average occupation of the corresponding lead, i.e., $I_{\eta}=-e\left\langle d \hat{n}_{\eta} / d t\right\rangle$ where $\hat{n}_{\eta}=\sum_{k \sigma} \hat{a}_{\eta k \sigma}^{\dagger} \hat{a}_{\eta k \sigma}$ is the number operator for the lead $F_{\eta}$. The current flowing in $F_{1}$ may be written as

$$
\begin{aligned}
I_{1}= & \frac{e}{h} \int\left[T_{A R, 11}\left(f_{1}-\bar{f}_{1}\right)+T_{A R, 12}\left(f_{1}-\bar{f}_{2}\right)\right. \\
& \left.+T_{12}\left(f_{1}-f_{2}\right)\right] d E
\end{aligned}
$$

where $f_{1}=f\left(E-e V_{1}\right)$ and $f_{2}=f\left(E-e V_{2}\right)$ are the corresponding Fermi distributions for electrons in the leads $F_{1}$ and $F_{2}$ and $\bar{f}_{1}=f\left(E+e V_{1}\right)$ and $\bar{f}_{2}=f\left(E+e V_{2}\right)$ are the corresponding ones for holes. By comparing the Fermi distributions one is able to determine each contribution in Eq. (8). In fact, $T_{12}$ is the co-tunneling current of electrons from $F_{1}$ to $F_{2}$ through the $Q D ; T_{A R, 11}$ accounts for the Andreev reflection in $F_{1}$ and finally $T_{A R, 12}$ is the crossed Andreev reflection of an electron from $F_{1}$ as a hole in $F_{2}$. The explicit transmittance expressions for each process may be found in Refs. [23,45].

The corresponding equation for $I_{2}$ can be obtained from $I_{1}$ just replacing the $1 \rightarrow 2$ in the previous equations. We point out that the expression for the current is the same as obtained by Y. Zhu et al. [23] for a non-interacting QD. In the present case, in spite from the fact of the current formula resembles the one obtained in Ref. [23], it is being considered the presence of interactions into the QD which means that matrix elements of the Green's functions must be determined in a self-consistent calculation. However, it was used a Hubbard-I approximation to tackle the electronic correlations within the QD which allows us to obtain a Landauer-like equation for the current. This is an advantage in the sense that one can obtain analytic expressions for the transmittances $T_{12}, T_{A R, 11}$ and $T_{A R, 12}$.

\section{Results and discussion}

In the following results, we consider the Andreev regime in which the applied bias range is bounded by the superconductor energy gap. Since this quantity is the natural energy scale of the problem, all the physical parameters are presented in units of the superconductor gap.

Before proceeding to the results, a brief discussion about the validity of the approximation used to treat the Coulomb correlations is in order. The decoupling scheme used to close the system of equations does not describe the so-called Kondo effect in QDs that appears at low temperatures [46]. However, in the present case, the high polarization values suppress the Kondo effect $[45,46]$ and the use of Hubbard-I approximation is completely justified in this case $[47,48]$.
The superconductor gap of a common superconductor such as aluminum [49] is given by $\Delta_{\mathrm{Al}}=0.34 \mathrm{meV}$. By using $k_{\mathrm{B}} T=0.5 \Delta_{\mathrm{Al}}$ one obtains $T \simeq 1.95 \mathrm{~K}$. Notice that the value of $U=0.85$ was fixed in order to comply with the weak tunneling regime in which the ratio $U / \Gamma \gg 1$. In fact, with the values used we obtain $U / \Gamma=$ 85 which is of the same order of magnitude as the ratio used in Ref. [26].

Sothmann et al. [28] address the effect of the exchange field induced on the QD by studying the electrical current entering into the superconductor. It was considered a strong coupling to the superconductor $\left(\Gamma_{s}=0.40 U\right)$ and bias voltages up to $\mu / U \simeq 1.5$. In contrast, we have used a fixed bias voltage $e V_{1}=0.07 \Delta$ applied to $F_{1}$ which leads to $e V_{1} / U \simeq 0.08$ for $U=0.85 \Delta$. In addition, the temperature used in Ref. [28] was $k_{B} T=0.01 U$ while in the present work the values $k_{B} T=0.5 \Delta$ and $U=0.85 \Delta$ yield $k_{B} T \simeq 0.59 U$. The comparison of these parameters allows us to safely neglect the effects of an exchange field induced on the QD. Therefore, the approximation used in this work yields realistic results that might be probed with the present technology.

Notice that all the results to be discussed were determined by using fixed polarization values. For the ferromagnet $F_{1}$ we have used $P_{1}=0.91$ while $F_{2}$ was assumed to be full polarized, $P_{2}=1$.

\subsection{Zero-bias regime}

Here we consider the transmittance curves at zero-bias voltage for both co-tunneling between the ferromagnet as well as the direct Andreev reflection in $F_{1}$. In order to make easier to visualize the lineshapes for these curves, we have normalized these curves by their maximum values. The high polarization values strongly reduce their maximum values which make difficult to observe the detailed variation with the external variables.

We start considering the tunneling between the ferromagnets, characterized by the transmittance $T_{12}$. In Fig. 2, it is shown the contour plot of $T_{12}$ as a function of the electron energy within the $\mathrm{QD}, \varepsilon$, and the gate voltage, $V_{g}$.

The presence of electronic correlations within the QD introduces two resonances located at $\varepsilon$ and $\varepsilon+U$ located by two diagonal lines in the contour plot. These resonances may better visualized in Figs. 2(b) and 2(c) in which some profiles labeled as A1, B1, C1 and D1 are shown. This pattern is similar of an interacting QD coupled to two normal metal reservoirs; the coupling to the superconductor is not evident from Fig. 2(a). This is a result of the energy scale since the resonances are resolved in the scale of the coupling constants $\Gamma_{1}$ and $\Gamma_{2}$ which are set to $0.005 \Delta$. In this way, in Fig. 2(d) the contour plot within this scale is shown where the effect of the coupling to the superconductor can be observed. In fact, the contour plot is divided into four regions in which the transmittance can reach a maximum for particular values of gate voltage and energy. The curves labeled as B2 and C2 shown in Figs. 2(e) and 2(f), respectively, reveal a two dip structure in transmittance with a central well defined peak.

Such a central peak is the main result of this work revealing the effect of the coupling to the superconductor; without its presence, $T_{12}$ is characterized by a single central dip due to the interference between the different spin channels. The coupling to the superconductor changes dramatically the profile of $T_{12}$ with a resonance in the place of dip, i.e., the presence of the superconductor improves the transport between the ferromagnets. This is a non-trivial effect resulting from interplay between the Andreev bound states and the spin polarization from the ferromagnets. We have called this effect as a Fano-like interference since it is a similar behavior observed in other nanostructures composed by double QDs [32]. It is worth mentioning that the misalignment between the magnetizations of $F_{1}$ and $F_{2}$ is also crucial for the phenomenon to emerge. In fact, in Fig. 2(e) the green-dotted curve was calculated for the same pa- 

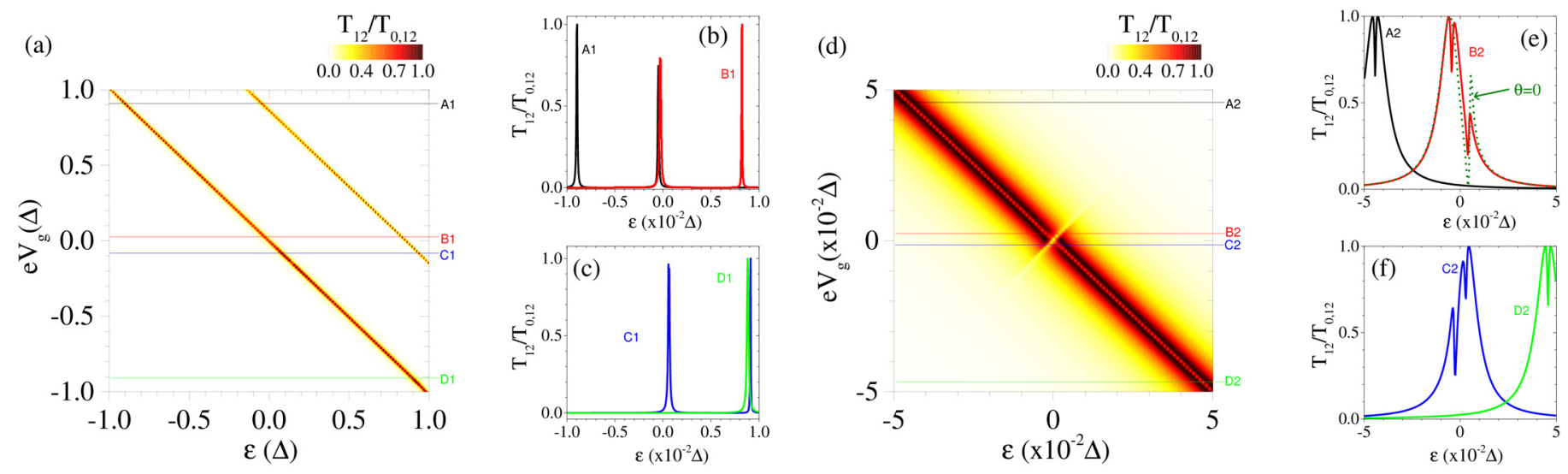

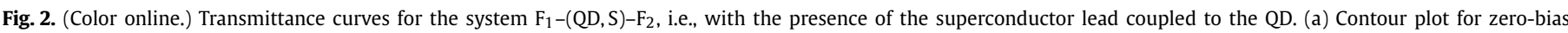

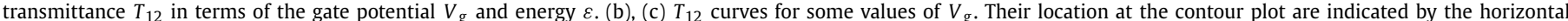

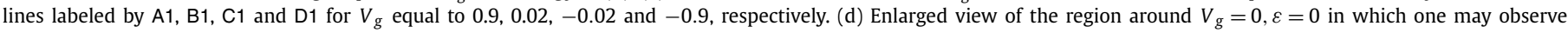

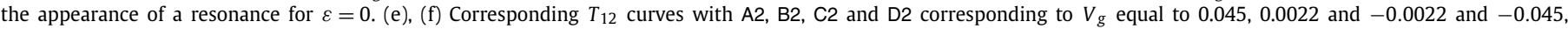

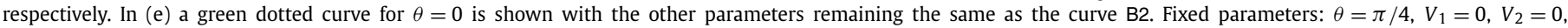
$\Gamma_{1}=\Gamma_{2}=0.005, \Gamma_{s}=0.01, P_{1}=0.91, P_{2}=1.0, U=0.85$ and $k_{B} T=0.5$. All the parameters are scaled by the energy gap of the superconductor lead.
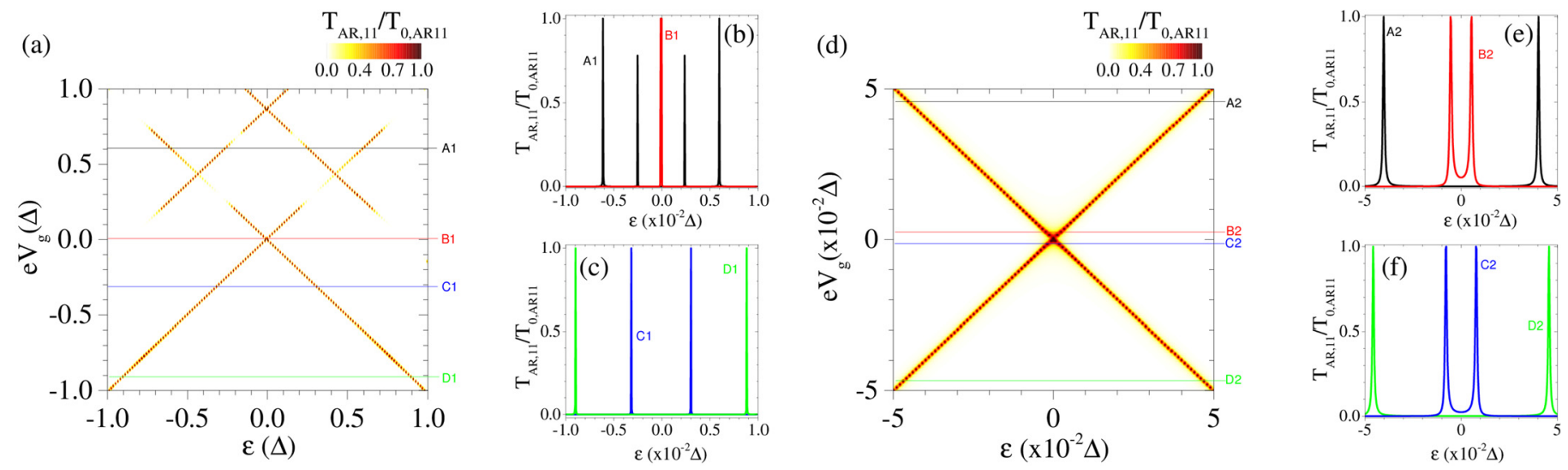

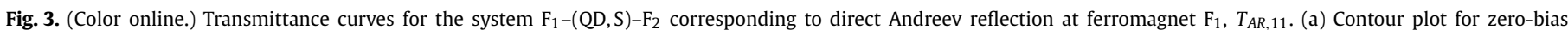

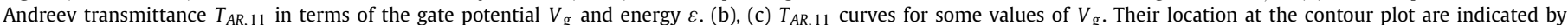

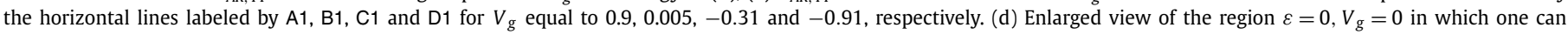

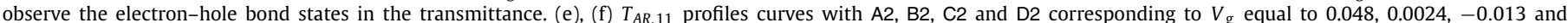

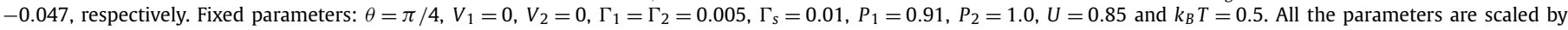
the energy gap of the superconductor lead.

rameters as the curve $\mathrm{B} 2$, with the exception of $\theta$, which was set to 0 . The additional resonance does not emerge in this case. The effect only appears for intermediate values of $\theta$ from $\theta \sim 0.07 \pi$ up to $\sim 0.85 \pi$.

To make evident the role of the superconductor on this effect, in Fig. 3(a) we show the corresponding direct Andreev transmittance $T_{A R, 11}$ normalized by its maximum value. The signature of the Andreev bound states is the two peak structure corresponding to electron and hole states located to $\varepsilon$ and $-\varepsilon$, respectively. The electronic correlations split these states as evident in the profile A1 of Fig. 3(b). As the gate voltage is changed, the resonance conditions are modified and the spectrum changes from four peaks to just one peak at $V_{g}=0$. For negative values of $V_{g}$ the transmittance just present a two peak structure. Within the scale of $\Gamma$, one can notice two crossing lines in the contour plot of Fig. 3(d) corresponding to two resonances as shown in Figs. 3(e) and 3(f). By comparing the contour plots of Fig. 3(d) to the corresponding one of Fig. 2(d), it is clear that these are complementary patterns of resonances: in the regions at which the $T_{A R, 11}$ exhibits a maximum value, the transmittance $T_{12}$ presents a dip. Thus, the coupling to the superconductor results in a "leakage" of states from the direct channel between the ferromagnets to Andreev states. This leads to the pattern observed in Fig. 2. It is worth mentioning that Calle et al. [32] have studied a three-terminal nanostructure composed by two normal metals coupled by a double QD system and a superconductor. In this system they have observed a similar pattern as shown in Fig. 2. The authors attributed such a feature to the Fano effect due to interference between two electronic paths. Here, the origin of such a pattern is different being related to the interplay between the Andreev bound states and spin polarization provided by the ferromagnets. The result is the leakage of electrons to the superconductor when the QD energy meet the Andreev resonances.

\subsection{Finite-bias regime}

In the finite bias regime, Coulomb correlations couple the transmittance and spectral properties of the QD to the bias applied to $\mathrm{F}_{1}$ and $\mathrm{F}_{2}$. In this way, for each value of $V_{1}$ and $V_{2}$ there is a corresponding transmittance curve. In spite of these modifications, it is also possible to recognize the signatures of Fano-like interference in the non-equilibrium case.

In order to illustrate such an effect, in Fig. 4 the current as a function of $e V_{g}$ is shown for direct co-tunneling between the leads as well as direct and crossed AR. A finite bias $e V_{1}=0.07$ is applied to $F_{1}$ while the superconductor and the ferromagnet $F_{2}$ are kept grounded. The other parameters are the same as used in 

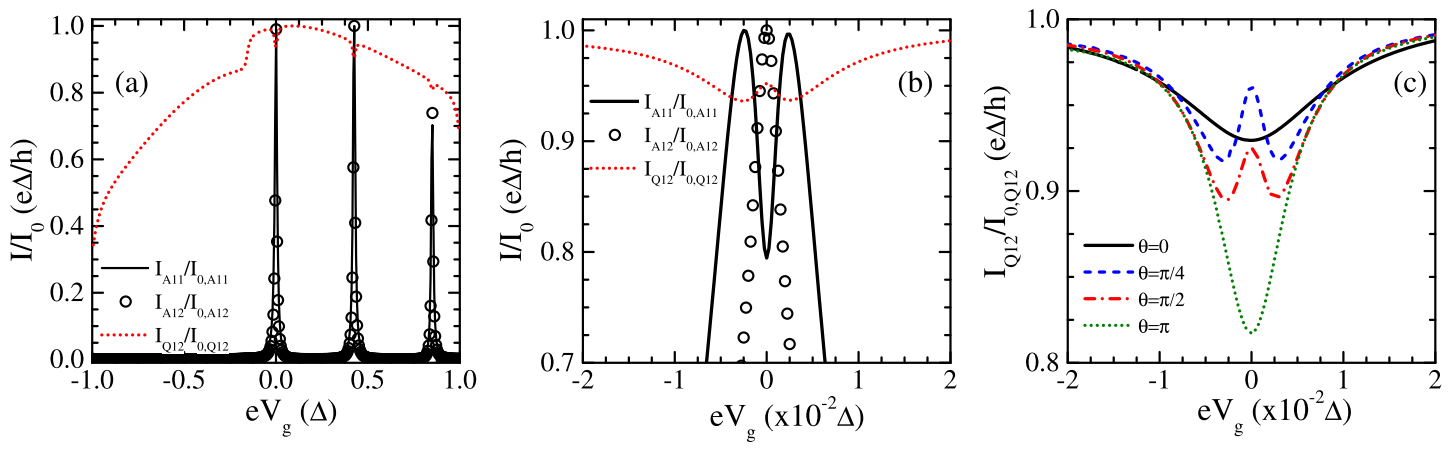

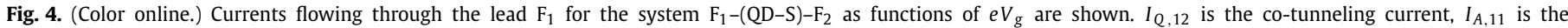

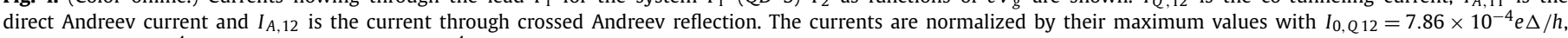

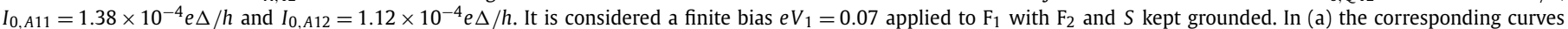

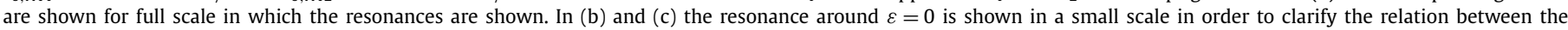

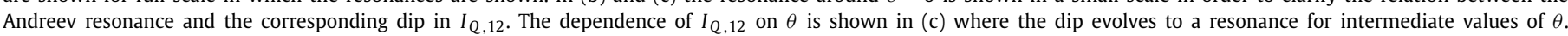

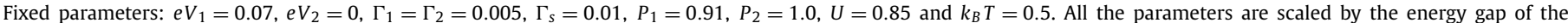
superconductor lead.

Figs. 2 and 3. Notice also that the currents are normalized to its maximum value in order to compare their behavior. In fact, the high polarization values strongly reduce the current by direct AR while the crossed AR increases with $\theta$ being the dominant mechanism of transport for $\theta=\pi$.

In Fig. 4(a) the currents for both direct and crossed AR display similar behaviors with three peaks located at $e V_{g}=0, e V_{g}=U / 2$ and $e V_{g}=U$. This splitting is a result of the electronic correlations within the QD. The current $I_{Q 12}$ between the ferromagnets exhibits a series of dips corresponding to the Andreev resonances. As discussed in the zero-bias results, the effect of the coupling to the superconductor is better understood by looking at the scale set by the constant couplings $\Gamma_{1}$ and $\Gamma_{2}$. In fact, as shown in Fig. 4(b), the region around $e V_{g}=0$ displays a splitting of the direct Andreev current (solid black curve) with a central dip located at $e V_{g}=0$; the current $I_{Q, 12}$ exhibits a corresponding peak at $e V_{g}=0$ with two dips located at the same place as the Andreev resonances. In this way, the currents keep the same complementary behavior as presented by the zero-bias transmittances. In contrast, the crossed AR current presents a single peak at $e V_{g}=0$ as shown in Fig. 4(b). The reason is that the crossed AR involves both ferromagnetic leads which work in a coherent way: an electron of $F_{1}$ combines with a second electron of $F_{2}$ in order to form a Cooper pair into the superconductor. As a result, the dependence of this current on the QD spectral properties is different in comparison to direct AR as well as the cotunneling current $I_{Q, 12}$. This point has been discussed in depth in Ref. [31]. When the orientation angle $\theta$ is changed from 0 to $\pi$ the crossed AR dominates the transport and the current is fully carried by crossed AR for full polarization.

The dependence of the current $I_{Q, 12}$ on the gate voltage for some values of the angle $\theta$ is shown in Fig. 4(c). It can be noted that this current shows a central peak for intermediate values of $\theta$ which are suppressed for $\theta=0$ and $\pi$. This is the signature of the Fano-like interference in the nonequilibrium case, resulting from the interference between different spin orientations as well as the Andreev bound states induced in the QD.

\section{Conclusion}

In this work, we have studied the interference effects on $F_{1}-(Q D, S)-F_{2}$ system due to the coupling to a conventional superconductor. The interplay between spin imbalance and Andreev bound states gives rise to a central resonance in the transmittance $T_{12}$, accounting for the transport between the ferromagnets. By comparing $T_{12}$ and direct AR transmittance $\left(T_{A, 11}\right)$, it is observed a complementary relation between these two channels of conduction. This relation allows one to probe the effects of the Andreev bound states in the transport between two ferromagnets. In the non-equilibrium situation, it is also possible to observe the signatures of such interference effects in the electrical current as shown in Fig. 4. It is worth mentioning that the correlation effect is to modify the signatures of the Fano-like effect and they do not kill the effect. The results presented may be reproduced in experiments by using half-metal ferromagnets. Additionally, high polarization ( $>90 \%$ ) values have been obtained in ferromagnetic films of $\mathrm{CrO}_{2}$ by Soulen Jr. and co-workers [50]; polarization values over $85 \%$ have been reported in ferromagnetic semiconductors based on GaMnAs [51]. Hence, the results above presented are realistic and may be implemented with the state-of-art of experiments.

\section{Acknowledgements}

P.O. acknowledges the support of FONDECYT grant 114057, DGIP/USM internal grant 11.14.68 and CONICYT ACT 1204.

\section{References}

[1] Z. Chen, B. Wang, D.Y. Xing, J. Wang, A spin injector, Appl. Phys. Lett. 85 (2004) 2553-2555.

[2] X. Cao, Y. Shi, X. Song, S. Zhou, H. Chen, Spin-dependent Andreev reflection tunneling through a quantum dot with intradot spin-flip scattering, Phys. Rev. B 70 (2004) 235341

[3] J.-F. Feng, S.-J. Xiong, Tunneling resonances and Andreev reflection in transport of electrons through a ferromagnetic metal/quantum dot/superconductor system, Phys. Rev. B 67 (2003) 045316.

[4] Y. Zhu, Q.-f. Sun, T.-h. Lin, Andreev reflection through a quantum dot coupled with two ferromagnets and a superconductor, Phys. Rev. B 65 (2001) 024516.

[5] F. Dolcini, L. Dell'Anna, Multiple Andreev reflections in a quantum dot coupled to superconducting leads: effect of spin-orbit coupling, Phys. Rev. B 78 (2008) 024518 .

[6] Intradot spin-flip Andreev reflection tunneling through a ferromagnetquantum dot-superconductor system with AC field, Phys. Lett. A 372 (2008) 6773-6777.

[7] J.P. Morten, A. Brataas, W. Belzig, Circuit theory of crossed Andreev reflection, Phys. Rev. B 74 (2006) 214510.

[8] C. Benjamin, Crossed Andreev reflection as a probe for the pairing symmetry of ferromagnetic superconductors, Phys. Rev. B 74 (2006) 180503.

[9] R. Mélin, S. Peysson, Crossed Andreev reflection at ferromagnetic domain walls, Phys. Rev. B 68 (2003) 174515.

[10] F. Giazotto, F. Taddei, F. Beltram, R. Fazio, Crossed Andreev reflection-induced magnetoresistance, Phys. Rev. Lett. 97 (2006) 087001.

[11] G. Deutscher, Crossed Andreev reflections, J. Supercond. 15 (2002) 43-47.

[12] R. Mélin, Crossed conductance in ferromagnet/superconductor/ferromagnet double junctions: role of out-of-equilibrium populations, Phys. Rev. B 72 (2005) 054503.

[13] C. Benjamin, R. Citro, Nonlocal pure spin current injection via quantum pumping and crossed Andreev reflection, Phys. Rev. B 72 (2005) 085340. 
[14] L. Bai, Z.-Z. Zhang, L. Jiang, F.-R. Tang, Spin accumulation and spin-dependent Andreev reflection current in a T-shaped double quantum dots, Physica E, LowDimens. Syst. Nanostruct. 43 (2010) 446-451.

[15] L. Bai, R. Zhang, C.-L. Duan, Andreev reflection tunneling through a triangular triple quantum dot system, Physica B, Condens. Matter 405 (2010) 4875-4880.

[16] R. Zhang, X.-F. Peng, K. Han, Y.-F. Shen, F.-R. Tang, L. Bai, Spin-dependent Andreev reflection in a three-terminal Aharonov-Bohm interferometer with coherent indirect coupling, Eur. Phys. J. B 86 (2013).

[17] G. Michałek, B.R. Bułka, T. Domański, K.I. Wysokiński, Interplay between direct and crossed Andreev reflections in hybrid nanostructures, Phys. Rev. B 88 (2013) 155425.

[18] K. Bocian, W. Rudzinski, Influence of intradot Coulomb correlations on the Andreev reflection in a ferromagnetic metal/quantum dot/superconductor hybrid junction, Eur. Phys. J. B 86 (2013).

[19] P. Trocha, J. Barnaś, Spin-polarized Andreev transport influenced by Coulomb repulsion through a two-quantum-dot system, Phys. Rev. B 89 (2014) 245418.

[20] C.M. Varma, Y. Yafet, Magnetic susceptibility of mixed-valence rare-earth compounds, Phys. Rev. B 13 (1976) 2950-2954.

[21] J. Hubbard, Electron correlations in narrow energy bands, Proc. R. Soc., Math. Phys. Eng. Sci. 276 (1963) 238-257.

[22] Y.V. Nazarov, Y.M. Blanter, Quantum Transport: Introduction to Nanoscience, Cambridge University Press, 2009.

[23] Y. Zhu, Q.-f. Sun, T.H. Lin, Andreev reflection through a quantum dot coupled with two ferromagnets and a superconductor, Phys. Rev. B 65 (2001) 024516.

[24] J.-F. Feng, X.-S. Wu, S.-S. Jiang, Tunneling resonances and Andreev reflection through an interaction quantum dot coupled with two half-metals and a superconductor, J. Appl. Phys. 99 (2006).

[25] K.P. Wójcik, I. Weymann, Proximity effect on spin-dependent conductance and thermopower of correlated quantum dots, Phys. Rev. B 89 (2014) 165303.

[26] I. Weymann, P. Trocha, Superconducting proximity effect and zero-bias anomaly in transport through quantum dots weakly attached to ferromagnetic leads, Phys. Rev. B 89 (2014) 115305.

[27] D. Futterer, M. Governale, M.G. Pala, J. König, Nonlocal Andreev transport through an interacting quantum dot, Phys. Rev. B 79 (2009) 054505.

[28] B. Sothmann, D. Futterer, M. Governale, J. König, Probing the exchange field of a quantum-dot spin valve by a superconducting lead, Phys. Rev. B 82 (2010) 094514.

[29] M. Colci, K. Sun, N. Shah, S. Vishveshwara, D.J. Van Harlingen, Anomalous polarization-dependent transport in nanoscale double-barrier superconductor/ferromagnet/superconductor junctions, Phys. Rev. B 85 (2012) 180512.

[30] K. Sun, N. Shah, S. Vishveshwara, Transport in multiterminal superconductor/ferromagnet junctions having spin-dependent interfaces, Phys. Rev. B 87 (2013) 054509.

[31] E.C. Siqueira, G.G. Cabrera, Magnetoresistance and transistor-like behavior of a double quantum-dot via crossed Andreev reflections, J. Appl. Phys. 111 (2012) 113905.

[32] A. Calle, M. Pacheco, P. Orellana, Fano effect and Andreev bound states in T-shape double quantum dots, Phys. Lett. A 377 (2013) 1474-1478.
[33] Z.Y. Zeng, B. Li, F. Claro, Electronic transport in hybrid mesoscopic structures: a nonequilibrium Green function approach, Phys. Rev. B 68 (2003) 115319.

[34] Z. Zeng, B. Li, F. Claro, Electron transport in interacting hybrid mesoscopic systems, Eur. Phys. J. B 32 (2003) 401-405.

[35] P. Fazekas, Lecture Notes on Electron Correlations and Magnetism, World Scientific, Singapore, 1999.

[36] J. Bardeen, L.N. Cooper, J.R. Schrieffer, Microscopic theory of superconductivity, Phys. Rev. 106 (1957) 162-164.

[37] F.S. Bergeret, A.F. Volkov, K.B. Efetov, Odd triplet superconductivity and related phenomena in superconductor-ferromagnet structures, Rev. Mod. Phys. 77 (2005) 1321-1373.

[38] K. Sun, C.-K. Chiu, H.-H. Hung, J. Wu, Tuning between singlet, triplet, and mixed pairing states in an extended Hubbard chain, Phys. Rev. B 89 (2014) 104519.

[39] M. Eschrig, T. Lofwander, Triplet supercurrents in clean and disordered halfmetallic ferromagnets, Nat. Phys. 4 (2008) 138-143.

[40] T. Klapwijk, Magnetic nanostructures: supercurrents in ferromagnets, Nat. Phys. 6 (2010) 329-330.

[41] R.S. Keizer, S.T.B. Goennenwein, T.M. Klapwijk, G. Miao, G. Xiao, A. Gupta, A spin triplet supercurrent through the half-metallic ferromagnet $\mathrm{CrO}_{2}$, Nature 439 (2006) 825-827.

[42] J. Rammer, H. Smith, Quantum field-theoretical methods in transport theory of metals, Rev. Mod. Phys. 58 (1986) 323-359.

[43] H. Haug, A.P. Jauho, Quantum Kinetics in Transport and Optics of Semiconductors, Springer, Berlin, 1996.

[44] J. Fransson, Non-Equilibrium Nano-Physics: A Many-Body Approach, Lecture Notes in Physics, Springer, 2010.

[45] E.C. Siqueira, P.A. Orellana, A.C. Seridonio, R.C. Cestari, M.S. Figueira, G.G. Cabrera, Interference effects induced by Andreev bound states in a hybrid nanostructure composed by a quantum dot coupled to ferromagnetic and superconductor leads, arXiv:1409.5390, 2014.

[46] D. Goldhaber-Gordon, H. Shtrikman, D. Mahalu, D. Mahalu, D. Abusch-Magder, U. Meirav, M.A. Kastner, Kondo effect in a single-electron transistor, Nature 391 (1998) 156-159.

[47] J. Martinek, Y. Utsumi, H. Imamura, J. Barnaś, S. Maekawa, J. König, G. Schön, Kondo effect in quantum dots coupled to ferromagnetic leads, Phys. Rev. Lett. 91 (2003) 127203.

[48] J. Martinek, M. Sindel, L. Borda, J. Barnaś, J. König, G. Schön, J. von Delft, Kondo effect in the presence of itinerant-electron ferromagnetism studied with the numerical renormalization group method, Phys. Rev. Lett. 91 (2003) 247202.

[49] R. Parks, Superconductivity: Part 1, Superconductivity (In Two Parts), Taylor \& Francis, 1969.

[50] R.J. Soulen Jr., J.M. Byers, M.S. Osofsky, B. Nadgorny, T. Ambrose, S.F. Cheng, P.R. Broussard, C.T. Tanaka, J. Nowak, J.S. Moodera, A. Barry, J.M.D. Coey, Measuring the spin polarization of a metal with a superconducting point contact, Science 282 (1998) 85.

[51] J.G. Braden, J.S. Parker, P. Xiong, S.H. Chun, N. Samarth, Direct measurement of the spin polarization of the magnetic semiconductor (Ga, Mn)As, Phys. Rev. Lett. 91 (2003) 056602. 